\title{
Preclinical Alternative Model for Analysis of Porous Scaffold Biocompatibility Applicable in Bone Tissue Engineering
}

\author{
Eva Petrovova ${ }^{1}$, Maria Giretova 2 , Alena Kvasilova ${ }^{3}$, Oldrich Benada ${ }^{4}$, Jan Danko ${ }^{1}$, Lubomir Medvecky ${ }^{1,2}$ \\ and David Sedmera 3,5 \\ ${ }^{1}$ Institute of Anatomy, University of Veterinary Medicine and Pharmacy, Kosice, Slovak Republic; ${ }^{2}$ Institute of Materials Research, The Slovak Academy \\ of Sciences, Kosice, Slovak Republic; ${ }^{3}$ Institute of Anatomy, Charles University, Prague, Czech Republic; ${ }^{4}$ Institute of Microbiology, The Czech Academy \\ of Sciences, Prague, Czech Republic; ${ }^{5}$ Institute of Physiology, The Czech Academy of Sciences, Prague, Czech Republic
}

\begin{abstract}
Porous scaffolds represent a potential approach to repair critical-size bone defects. Vascularization is essential for bone formation and healing. This study establishes a method to monitor angiogenesis within porous biopolymer scaffolds made on the basis of polyhydroxybutyrate and chitosan. We used the chick and quail chorioallantoic membrane (CAM) assay as an alternative in vivo model to study the formation of new blood vessels inside the scaffold structure. The chemical properties of the biopolymer scaffold matrix surface were characterized as well as the tissue reaction of the CAM. Placing a piece of polymer scaffold on the CAM resulted in a vascular reaction documented visually and by ultrasound biomicroscopy. Histological analysis showed a myofibroblast reaction (smooth muscle actin-positive cells) without excessive collagen deposition. Cell invasion into the implant was observed and the presence of a vascular network was confirmed by identifying hemangioblasts and endothelial cells of quail origin using the QH1 marker. The CAM assay is a rapid and easy way to test biocompatibility and vasculogenic potential of new candidate scaffolds for bone tissue bioengineering while respecting the $3 R$ s.
\end{abstract}

\section{Introduction}

Regeneration maintains or renews the original tissue architecture. In case of damage or chronic degenerative disease, fibrotic repair occurs instead of the normal regenerative process. Such a response is considered a reparative process, since the replacement tissue neither contains the original cell types nor is the original tissue architecture reestablished (Stocum, 2002).

The term regenerative medicine is often used as a synonym for tissue engineering, though regenerative medicine focuses on using stem cells to form tissues, while tissue engineering makes use of a combination of cells, biochemical and physiochemical factors, and biomaterials to improve or replace biological functions (Langer and Vacanti, 1993). Many definitions of tissue engineering include a wide range of applications, however, in practice, tissue engineering closely focuses on repairing or replacing parts of or entire tissues (cartilage, bone, blood vessels, skin, muscle, nerves, etc.) including certain mechanical and structural properties that are required for proper functioning (Musumeci et al., 2014).
Angiogenesis is the physiological process of forming new capillaries from existing vessels. It is a tiered process involving activation of the existing endothelial cells, degradation of the extracellular matrix, proliferation and migration of endothelial cells, invasion of the stroma by the surrounding cells, and remodeling the extracellular matrix. Regulation of endothelial cell survival and migration strongly depends on the interaction of endothelial cells with extracellular matrix proteins via cell adhesion molecules, and the activities of growth factors and cytokines. Angiogenesis is characteristic of regeneration of normal tissues and hence is an important factor determining the safe and successful use of biomaterials in regenerative medicine (Chavakis and Dimmeler, 2002) as perfusion of the implant is needed to provide a feasible infrastructure upon which the new tissue can mature (Anderson et al., 2011). Induction of angiogenesis and subsequent development of a vascular bed in the engineered tissue is being actively pursued through combinations of physical and chemical cues, notably through the presentation of suitable topographies and growth factors (Klagsburn and Moses, 1999; Liu et al., 2012; Kant and Coulombe, 2018).
Received July 24, 2018; Accepted November 12, 2018; Epub November 23, 2018; (C) The Authors, 2018.

ALTEX 36(1), 121-130. doi:10.14573/altex.1807241

Correspondence: Eva Petrovova, DVM, PhD; Institute of Anatomy

University of Veterinary Medicine and Pharmacy,

Komenskeho 73, 04181 Kosice, Slovak Republic

(eva.petrovova@uvlf.sk)
This is an Open Access article distributed under the terms of the Creative Commons Attribution 4.0 International license (http://creativecommons.org/licenses/by/4.0/) which permits unrestricted use, distribution and reproduction in any medium, provided the original work is appropriately cited. 
Osteogenesis, the formation of bone, is closely connected with angiogenesis during the process of bone healing (Yu et al., 2017). One way to achieve a tissue-engineered bone tissue could be the use of an angiogenesis-promoting scaffold. Neovascularization into the implant not only supplies nutrients, oxygen, calcium, and phosphate, but also provides a transport route for the mesenchymal stem cells to facilitate bone regeneration (Stegen et al., 2015).

Scaffold that can promote osteogenesis and angiogenesis must have appropriate pore structure, biocompatibility, mechanical properties and processability to promote cell adhesion, migration, proliferation, and differentiation (Zhang et al., 2015). Chitosan is a hydrophilic biopolymer polysaccharide that induces and stimulates connective tissue rebuilding (Muzzarelli et al., 1988). It is a natural polymer with antibacterial properties that is biodegradable, biocompatible, highly abundant, and non-toxic. It can be molded into different forms (films, gels, sponges, fibers, nanoparticles, nanofibers) for diverse applications in tissue engineering (Concha et al., 2018; Poonguzhali et al., 2018). Because of the swelling of chitosan-based composites owing to water uptake during preparation, porous scaffold can be prepared by later lyophilization (Giretova et al., 2016). In bone tissue engineering, chitosan has frequently been used in combination with other bone-forming matrices such as hydroxyapatite or collagen (Tan et al., 2014). Poly-3-hydroxybutyrate (PHB) represents another natural, biodegradable and hydrophobic biopolymer that can be combined with chitosan or calcium phosphate to composites that are of interest for bone and cartilage regeneration and that may be suitable for the reconstruction of deeper defects.

The animal models play a key role in basic medical research. Advances in tissue engineering that lead to development of new therapeutic approaches are often based on in vitro experiments that are later followed by in vivo experiments. However, the use of rodents as model animals in experimental studies encounters ethical, practical and technical problems, which limit their use in certain areas of this research (Rashidi and Sottile, 2009). The avian embryo represents an easily available and cost-effective alternative model that may be used to test various biomaterials. Chicken or quail embryo development takes place outside the mother's body, obviating the need to sacrifice experimental animals or cause physical harm as is usually the case in implantation surgery. A further big advantage is that there is no need to apply for animal protocol approval for the chick/quail embryo in ovo as an experimental model as both are exempt from the horizontal legislation on the protection of animals used for scientific purposes in Europe (2010/63/EU), as well as applicable laws in the United States. The avian embryo, and especially its chorioallantoic membrane (CAM), thus provides a simple and effective alternative model to assess the biocompatibility of potential new bone implants (Kang et al., 2018; Tomco et al., 2017; Magnaudeix et al., 2016; Steffens et al., 2009) and can be considered a 3R method.

The CAM is used in many areas of research, including investigating molecules regulating the formation of blood vessels for use in the treatment of chronic inflammation, tumors, healing of wounds and fractures, drug delivery, and toxicological analysis (Hazel, 2003; Eun and Koh, 2004; Özcetin et al., 2013; Pandit et al., 2017; Ribatti, 2016). The CAM consists of two extra-embryonic membranes: the chorion and the allantois, which fuse together on embryonic day (ED) 4. Histologically, the CAM is comprised of three layers: the ectoderm, mesoderm, and endoderm. It serves as a respiratory and excretory organ with a non-innervated, transparent matrix and a vascular network (Tay et al., 2012). On ED8, the proliferation and differentiation of blood vessels is already well advanced, forming an extensive and easily accessible arteriovenous system composed of the umbilical arteries and veins (Djonov et al., 2000), which can serve as a surrogate blood supply for organ culture, and hence a platform for biomaterial testing (Borges et al., 2003; Moreno-Jiménez et al., 2017). Over 700 publications have used the chick embryo CAM as a model system to study angiogenesis. More rarely, the Japanese quail CAM has also been used successfully (Buríková et al., 2016). A particular benefit of the quail CAM is that the quail-derived endothelium expresses the unique marker QH1, which can be identified using a specific antibody (Pardanaud et al., 1987; Drake et al., 1997; Brown et al., 1999; Giles et al., 2005; Sedmera and McQuinn, 2008).

In recent years, the field of tissue engineering has accepted the CAM model as a useful, quick, and cheap alternative to the traditional animal models (rabbit ears, rodent skin, avascular cornea of the rabbit, cheek pouches of the hamster; Ribatti et al., 2006; Da-Lozzo et al., 2013; Vargas et al., 2013) to evaluate angiogenesis in new implants and weed out less promising scaffold candidates, thus reducing or replacing in vivo animal experiments (Falkner et al., 2004).

This study describes a new approach to detect the formation of blood vessels inside biopolymer scaffolds using the CAM of the developing avian embryo as an alternative to traditional mammalian models, which would make this model particularly attractive for the rapid biocompatibility screening of porous biomaterials for regeneration of hard tissues.

\section{Materials and methods}

\section{Preparation and characterization of composite scaffold}

Polyhydroxybutyrate/chitosan scaffolds (PHB/CHIT) were prepared according to the method of Medvecky et al. (2014). Briefly, polyhydroxybutyrate (GoodFellow, Cambridge, England) dissolved in propylene carbonate ( $1 \%$ solution of $\mathrm{PHB})$ and chitosan (Sigma Aldrich, middle, 1\% solution in 1\% acetic acid) were mixed together at a 1:1 ratio using a magnetic stirrer at $400 \mathrm{rpm}$. After $10 \mathrm{~min}$ of mixing, acetone (about $5 \mathrm{ml}$ ) was slowly added to the suspension to achieve precipitation of biopolymers. Final blends were filtered, washed with distilled water, and molded into a larger block $(4 \times 25 \times 1 \mathrm{~mm})$, which was then cut into smaller pieces with the final dimensions of $4 \times 4 \times 1 \mathrm{~mm}$ and lyophilized (Freeze dryer, ilShin Biobase Europe, Ede, The Netherlands) for $6 \mathrm{~h}$ (Fig. 1). Swelling of the composite samples was measured in $1.5 \mathrm{ml}$ vials by immersion of porous substrates (approximately $20 \mathrm{mg}$ ) in $0.9 \% \mathrm{NaCl}$ solution at $37^{\circ} \mathrm{C}$ up to a constant mass. Soaking was done in triplicate and swelling was evaluated as the ratio of wet weight to dry weight. The microstructure of the scaffolds was observed by scanning electron microscopy (FE SEM JEOL7000). The phase analysis of the blend was evaluated by X-ray powder diffraction analysis (XRD, Philips X Pert Pro). 

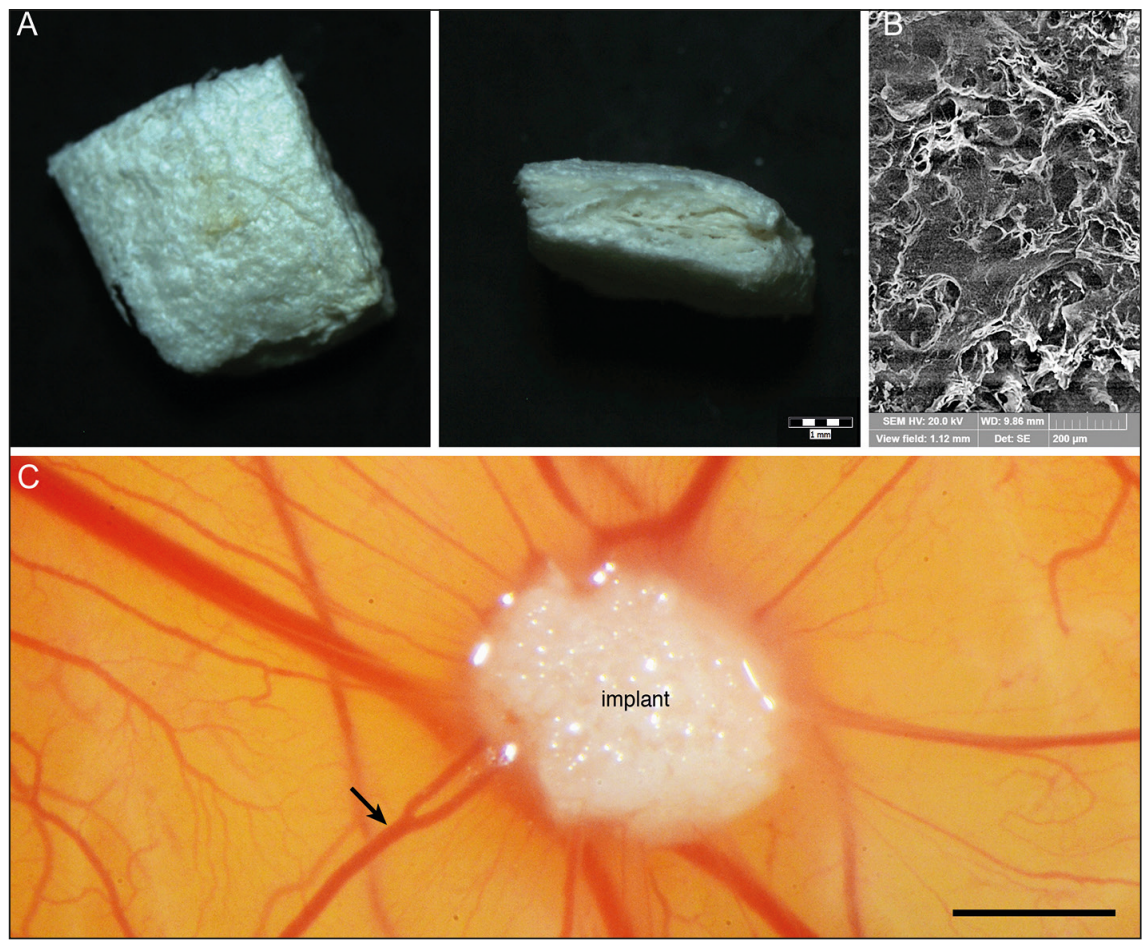

Fig. 1: Structure of PHB/CHIT scaffold and evidence of CAM blood vessels converging toward the implanted scaffold

(A) Macrostructure of PHB/CHIT scaffold, scale bar: $1 \mathrm{~mm}$. (B) Ultrastructure of $\mathrm{PHB} /$ CHIT scaffold with pores (SEM), scale bar: $200 \mu \mathrm{m}$. (C) Macroscopic evidence of CAM blood vessels converging toward the implanted PHB/CHIT scaffold (arrows) on ED10; scale bar: $1 \mathrm{~mm}$; 42 biological replicates (chick), 30 biological replicates (quail), for confirmatory images see ${ }^{1}$.

1 doi:10.14573/altex.1807241s1

\section{Cytotoxicity testing}

Cytotoxicity evaluation was carried out using MC3T3E1 mouse preosteoblasts (ECACC, Salisbury, UK) according to EN ISO 10993-5:2009. Cells were cultured in culture flasks in MEM (minimum essential medium) with Earl's balanced salts, $2 \mathrm{mM}$ L-glutamine (SAFC Biosciences, Hampshire, UK), 10\% fetal bovine serum (Sigma-Aldrich, St. Louis, MO, USA), and ATB-antimycotic (penicillin, streptomycin, amphotericin) solution (Sigma-Aldrich, St. Louis, MO, USA) at $37^{\circ} \mathrm{C}$ in $5 \% \mathrm{CO}_{2}$ at $95 \%$ humidity. After the cells reached about $80 \%$ confluence, they were harvested using $0.25 \%$ trypsin-EDTA (Sigma-Aldrich, St. Louis, MO, USA). Scaffold samples were sterilized in an autoclave at $121^{\circ} \mathrm{C}$. Cell viability in the presence of scaffold samples was examined using the MTS test (Cell titer 96 aqueous one solution cell proliferation assay, Promega, Madison, WI, USA). The sterilized scaffolds (Ø $6 \mathrm{~mm}$, thickness $1 \mathrm{~mm}, \mathrm{n}=5$ ) were placed into the plate (treated 96-well tissue culture plate, cellGrade Brand, Wertheim, Germany), seeded with $1.0 \times 10^{4} \mathrm{MC} 3 \mathrm{~T} 3 \mathrm{E} 1$ mouse preosteoblasts in $200 \mu$ complete osteogenic medium alpha modification MEM with $50 \mu \mathrm{g} / \mathrm{ml}$ ascorbic acid, $50 \mathrm{nM}$ dexamethasone, and $10 \mathrm{mM}$ $\beta$-glycerophosphate (Sigma Aldrich, St. Louis, MO, USA) and cultured at $37^{\circ} \mathrm{C}$ in $5 \% \mathrm{CO}_{2}$ at $95 \%$ humidity. Cell viability in the presence of scaffolds was evaluated 2 and 10 days after cell seeding by measuring formazan concentrations produced by metabolically active cells in the culture medium using a UV-VIS spectrophotometer (Shimadzu, Griesheim, Germany) at a wavelength of $490 \mathrm{~nm}$. The mean measured absorbance of medium from wells with cell-seeded substrates was compared with the absorbance of medium from scaffold-free wells (negative control, $n=5$ ). The pure complete culture medium was used as a blank.
The attachment and morphology of cells were visualized by live/dead fluorescence staining using fluorescein diacetate/propidium iodide solution (green, live cells and red, dead cells) after $48 \mathrm{~h}$ of cell cultivation in the presence of scaffolds by an inverted optical fluorescence microscope (Leica DM IL LED, blue filter).

\section{Chick CAM ex ovo model for microscopical evaluation}

Avian embryos used in this study are exempt from Directive 2010/63/EU. Fertilized chicken hybrid eggs (Ross 308; n = 14) were purchased from the chicken farm of the Institute of Molecular Genetics (Koleč, Czech Republic) and delivered via courier in a temperature-controlled manner to ensure egg viability and quality. The eggs were incubated horizontally in a forced-draft constant-humidity incubator at $37.5^{\circ} \mathrm{C}$ and $60 \%$ relative humidity. At ED3 the eggshell was disinfected with $70 \%$ ethanol, then cracked and the egg content with chick embryo was carefully transferred into a hexagonal plastic weighing boat stored in a Petri dish partially filled with sterilized distillated water to maintain humidity, and incubated until ED6 in a still draft incubator $\left(37.5^{\circ} \mathrm{C}, 70 \%\right.$ relative humidity, without rocking; McQuinn et al., 2007).

On ED6, a piece $(2 \times 2 \times 1 \mathrm{~mm})$ of the sterilized porous scaffold (CHIT/PHB) was gently placed on the chorioallantoic membrane ex ovo using suture tying forceps.

For visual evaluation of vascular density and video blood flow observation into/outside the scaffold we used a stereomicroscope (Leica MZ125) fitted with a DSLR camera (D7000, Nikon, Tokyo, Japan) for video documentation on ED10. Blood contrast was enhanced using a green interference filter inserted into a KLD250 halogen light source. Ultrasound biomicroscopy examination of implants was performed ex ovo on a high-resolution imaging system Vevo 770 (VisualSonics, Toronto, Canada) using 
B-Mode imaging (scanhead \#708), which allows the acquisition of two-dimensional images of the vessels inside of scaffolds as well as blood flow monitoring in a temperature-controlled setup (McQuinn et al., 2007).

\section{Quail CAM in ovo model for evaluation of angiogenesis and biocompatibility}

For implantation of scaffolds on the quail CAM in ovo, we used a method modified from Ribatti et al. (2006). Fertilized Japanese quail (Coturnix japonica) eggs $(\mathrm{n}=10)$ were purchased from the animal farm (Kosice, Slovakia) and delivered via courier in a temperature-controlled manner to ensure egg viability and quality. The eggs were incubated with blunt end up in a forced-draft constant-humidity incubator at $37.5^{\circ} \mathrm{C}$ and $60 \%$ relative humidity with continuous rocking. At ED3 the eggs were windowed on the blunt end, and the inner shell membrane (membrana papyracea) was carefully removed. The windows were closed using insulation tape and returned to a still draft incubator.

On ED5, a sterilized porous scaffold (CHIT/PHB) was gently placed on the chorioallantoic membrane, and the opening was re-sealed with the insulation tape. On ED10, Dent's solution was applied directly onto the CAM with the scaffold and surrounding vessels for $10 \mathrm{~min}$ at room temperature. Subsequently, the implant was removed and processed for evidence of angiogenesis and biocompatibility with histological techniques.

\section{Scanning electron microscopy (SEM)}

SEM analysis was used to investigate whether CAM microvilli grow into the pores of the scaffold. This would evidence biocompatibility of the tested biomaterial. In ovo implants at ED10 were fixed with $1 \%$ glutaraldehyde and $2 \%$ formaldehyde in PBS for $24 \mathrm{~h}$ at $4^{\circ} \mathrm{C}$, rinsed three times with PBS, and then post-fixed in $1 \%$ osmium tetroxide. After further rinsing, the specimens were dehydrated with ethanol series, critical point dried with $\mathrm{CO}_{2}$, and mounted on aluminum stubs. After sputter coating with gold, they were observed under a Bruker scanning electron microscope.

\section{Histological examination}

Themonitoring of the implant reaction and biocompatibility with the surrounding CAM was carried out using routine H\&E/Alcian Blue staining, followed by scanning of the slides using a 10x objective on an Olympus slide scanner.

The presence of myofibroblasts and macrophages ( $\alpha$-SMA, collagen I and CD68, respectively) as well as proliferative activity of the cells within the implant (phosphohistone-3, Anti-H3S10p) was evaluated with immunohistochemical staining of chick embryos. Deparaffinized sections were blocked in normal goat serum $(1: 10)$ and in $1 \%$ bovine serum albumin (BSA) in PBS with $0.1 \%$ Triton-X (Sigma-Aldrich, St. Louis, USA) for $60 \mathrm{~min}$ at RT. For staining with CD68 antibody, collagen I, and anti-H3S10p, antigen retrieval was performed with $10 \mathrm{mM}$ citrate buffer $(\mathrm{pH} 6)$ in a microwave oven $(750 \mathrm{~W})$ for two cycles of 5 min each prior to immunostaining. Primary antibodies (monoclonal mouse antibody $\alpha$-SMA, 1:800, Sigma-Aldrich \# A2547; polyclonal rabbit anti-mouse antibody collagen type I, 1:500, MDbiosciences, Oakdale, USA; monoclonal mouse antibody CD68, 1:100, Abcam ab955, Cambridge, UK or polyclonal rabbit antibody anti-H3S10p,
1:100, Millipore, CA, USA) were applied overnight at $+4^{\circ} \mathrm{C}$. Negative controls were obtained by omission of the primary antibody. The sections were then washed in PBS, and TRITC-conjugated goat anti-mouse secondary antibody or goat anti-rabbit secondary antibody for collagen type I (1:200, Jackson ImmunoResearch Laboratory, West Grove, PA, USA) was applied for $90 \mathrm{~min}$ in the dark at RT. Together with the secondary antibody, wheat germ agglutinin (WGA) coupled with the Alexa 488 dye was applied at 1:100 concentration to detect fibrous extracellular matrix including collagen (Benes et al., 2011). After washing in PBS, the nuclei were counterstained with Hoechst 33258 (1:100,000 diluted in $0.1 \%$ Triton-X in distilled water, Sigma-Aldrich, St. Louis, MO, USA). The sections were washed with distilled water and dehydrated in ethanol series, cleared in xylene, and mounted in DEPEX (Electron Microscopy Sciences, Hatfield, PA, USA).

The presence of hemangioblasts and endothelial cells was investigated with QH1 staining. Deparaffinized sections of ten quail embryos with implants were blocked in normal goat serum (1:10) and in $1 \%$ bovine serum albumin (BSA) in PBS with $0.1 \%$ Triton-X (Sigma-Aldrich, St. Louis, MO, USA) for 60 min at RT. QH1 (monoclonal mouse antibody, 1:1000, DSHB, Iowa, USA) was then applied overnight at $+4^{\circ} \mathrm{C}$. Negative controls were obtained by omission of the primary antibody. The sections were washed in PBS and TRITCconjugated goatanti-mouse secondary antibody (Jackson ImmunoResearch Laboratory, West Grove, PA, USA) was applied for $4 \mathrm{~h}$ in the dark at RT. After washing in PBS, the nuclei were counterstained with Hoechst 33258 (1:100,000 diluted in 0.1\% Triton-X in distilled water, Sigma-Aldrich, St. Louis, MO, USA). The sections were washed with distilled water and dehydrated in ethanol series, cleared in xylene, and mounted in permanent medium.

Fluorescently stained sections were examined first on a widefield epifluorescence microscope (Olympus BX51) and then documented on a confocal microscope Olympus FV-1000 BX61 (Olympus, Tokyo, Japan). Images were assembled and labeled using Adobe Photoshop (version 8.0) using Wiley standards for digital image manipulation. Brightness and contrast were adjusted using the "Adjust Levels" command on the entire image, and "Unsharp Mask" filter was used where necessary to enhance sharpness. No other image manipulations were performed.

CAM tissue remodeling in relation to the implanted scaffold was further analyzed using picrosirius red staining according to Junqueira et al. (1979). This specific staining of extracellular collagen fiber deposition was combined with immunohistochemical staining of WGA coupled with the Alexa 488 dye (1:100), and the nuclei were counterstained with Hoechst 33258 (1:100,000 diluted in $0.1 \%$ Triton- $\mathrm{X}$ in distilled water). For histological and immunofluorescent studies, we used 4 sections per slide, 2 slides per sample.

\section{Results}

\subsection{Properties, microstructure and in vitro cytotoxicity of composite scaffolds}

A macroporous microstructure with high fractions of spherical pores, which did not exceed $80 \mu \mathrm{m}$ diameter, and irregular macropores, with diameters up to $150 \mu \mathrm{m}$, was observed in PHB/CHIT 


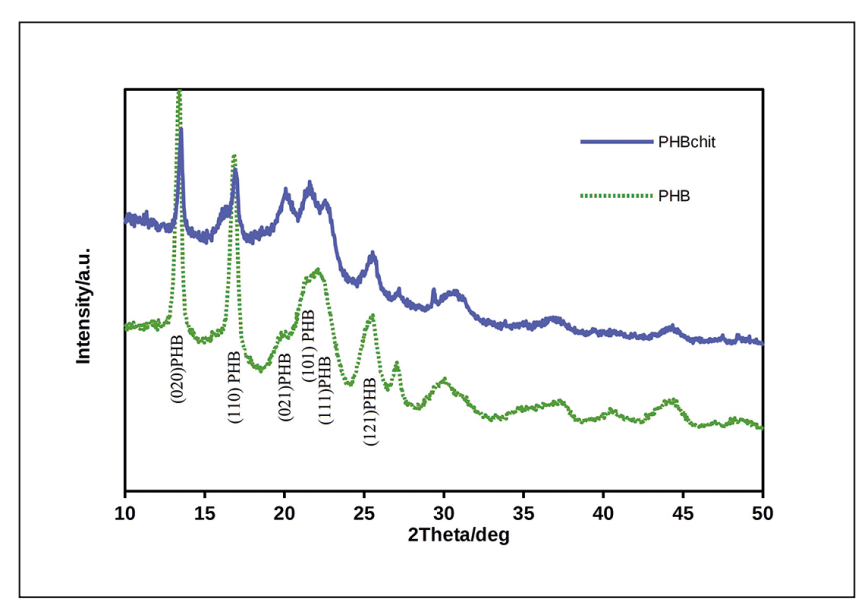

Fig. 2: XRD patterns of PHB and PHB/CHIT blend

Biological replicates (4).

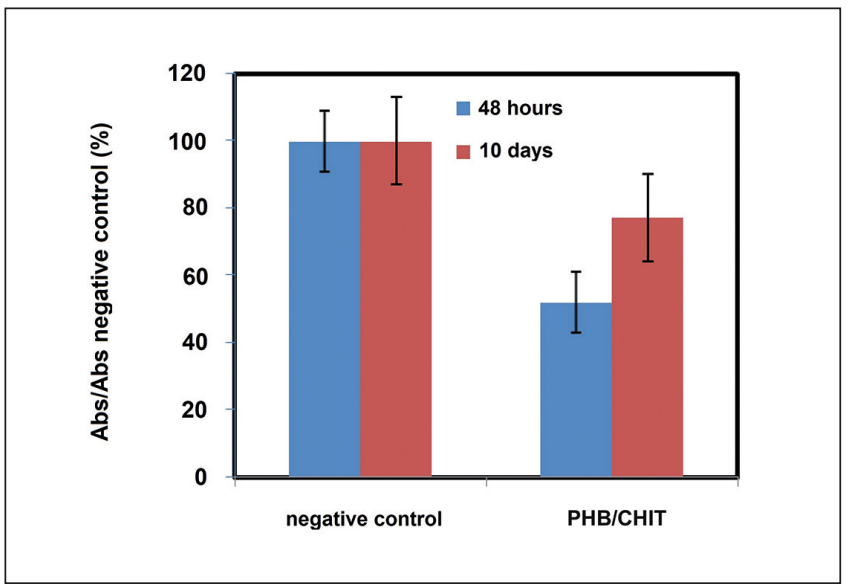

Fig. 3: Proliferation of osteoblasts on PHB/CHIT scaffolds in relation to negative control after 2 and 10 days of cell cultivation

Biological replicates (6), $p<0.01$ (one-way ANOVA, $\alpha=0.05$, StatMost statistical software).
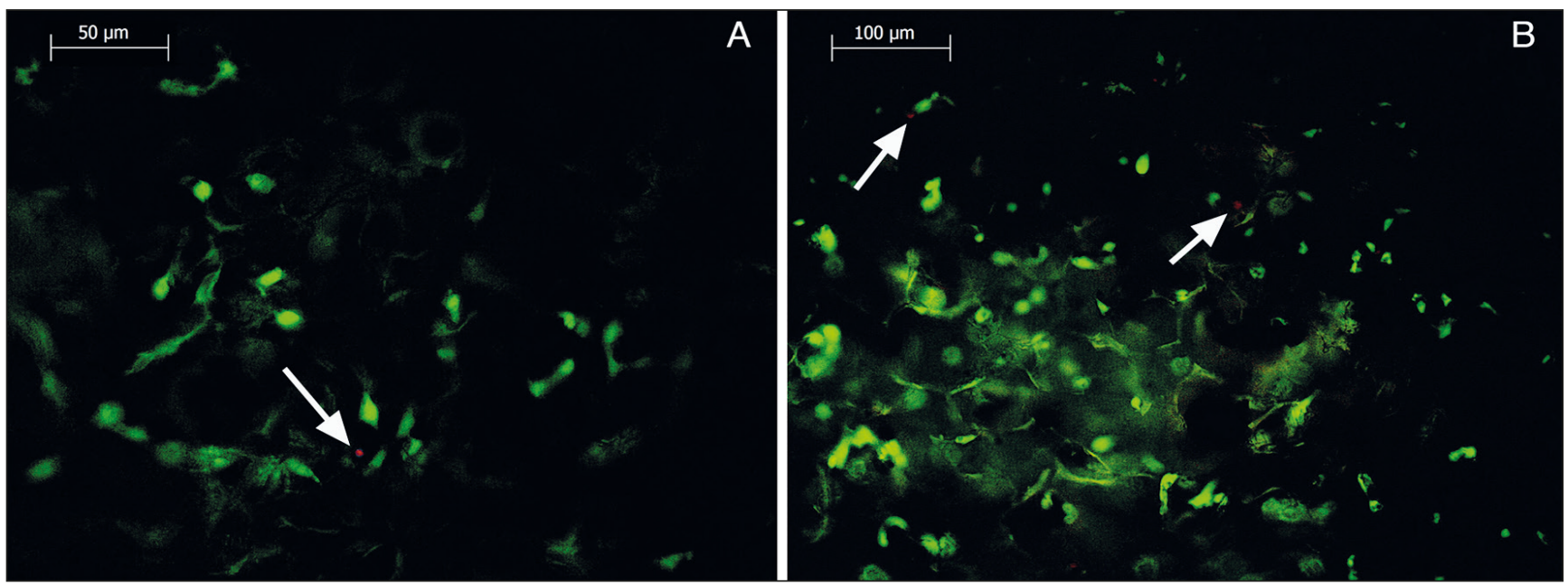

Fig. 4: Live/dead (arrows) fluorescence staining of cells growing on scaffolds after 2 days of cultivation

Biological replicates (9), technical replicates (45), for confirmatory images see ${ }^{3}$. Because of the porous structure of the material and nonstandard conditions for observation of cells by optical microscopy (especially flat surface), full images were not sharpened.

scaffolds (Fig. 1) by SEM. These pore sizes appear appropriate for migration of cells into the inner structure of the scaffolds to form specific tissues. No clearly distinguishable biopolymer fibers were visible in the detailed micrograph, which demonstrates uniform and homogeneous distribution of both biopolymers in the blend.

Dimensions of individual fibers have to be sufficient for crystallization into coherent regions, which was demonstrated by the XRD analysis (Fig. 2). The strong lines from reflections of (020), (110), (101), and (121) PHB planes verify a higher fraction of the crystalline PHB component in the PHB/CHIT blend contrary to the amorphous character of chitosan. A significant reduction of the average molecular mass of chitosan from $360 \mathrm{kDa}$ to about $41 \mathrm{kDa}$ was found after precipitation of biopolymers, which is probably the reason for low chitosan crystallinity.
A fast increase in scaffold mass was observed after the first $2 \mathrm{~h}$ of soaking when the mass increment achieved about $90 \%$ of the final water uptake. Constant mass of samples was reached after 2 days of swelling at which the dry mass of the biopolymer scaffold had more than doubled. This confirmed a highly hydrophilic character of the blend, which supports the adsorption of polar molecules (like nutrients, etc.) from the surrounding medium including body fluids.

Formazan production of preosteoblasts in contact with the scaffolds for 2 and 10 days of culture (results not shown) indicated an increase in the number of viable cells (about 7-fold), which demonstrates that the scaffolds support cell growth. The relative viability of cells increased from $50 \%$ after 2 days of culture to around $80 \%$ of the negative control after 10 days of culture, which confirms a non-cytotoxic behavior of biopolymer scaffolds (EN 

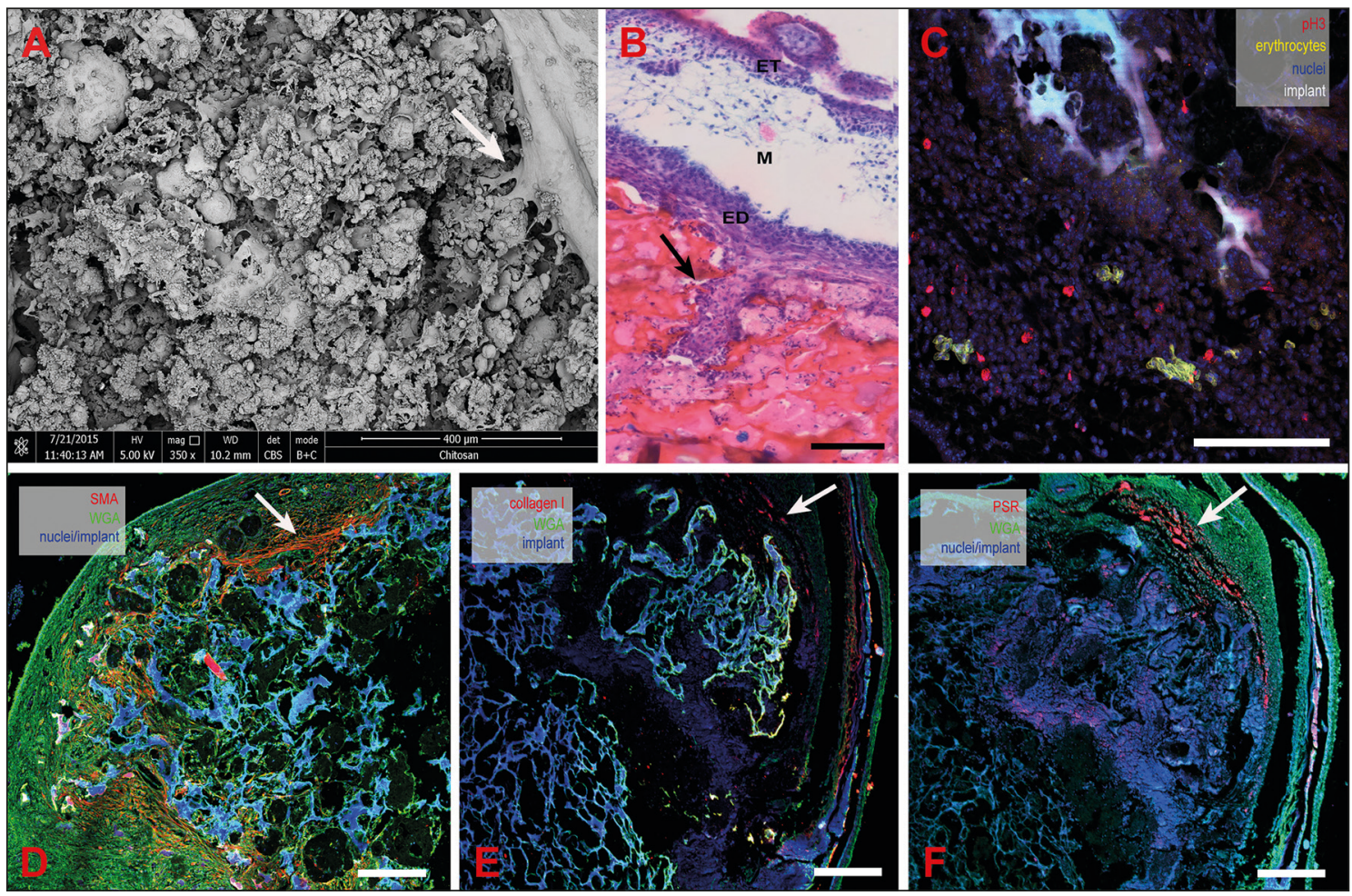

Fig. 5: Immunohistochemical analysis of the implant after 5 days in ovo

(A) Histology of the implant after 5 days in ovo. The formation of CAM villi growing into the implanted PHB/CHIT scaffold was observed using SEM as a comparison with histology (3D); scale bar: $400 \mu \mathrm{m}$. (B) Confirmation of CAM villi presence with H-E/Alcian blue staining; ET, ectoderm; M, mesoderm; ED, endoderm; scale bar: $100 \mu \mathrm{m}$. (C) Evidence of CAM tissue and proliferating cells within implanted scaffold; scale bar: $100 \mu \mathrm{m}$. (D) The presence of myofibroblasts on a border between scaffold and surrounding CAM tissue; scale bar: $100 \mu \mathrm{m}$. (E, F) Collagen was localized around implant without its excessive deposition; scale bar: $200 \mu \mathrm{m}$; biological replicates (15), technical replicates (720), for confirmatory images see1.

ISO 10993-5; Fig. 3). Images from live/dead fluorescence staining (Fig. 4) documented good adherence of cells to pore walls of scaffolds 2 days after seeding. The live cells' visible filopodia copied the pore walls and only few dead cells were observed (stained red; panel A, B of Fig. 4).

\subsection{Incorporation of scaffold into the CAM}

Five days after placing the PHB/CHIT scaffold on the CAM, it was well incorporated. Hyperplasia of the CAM tissue under the scaffold was observed in all cases. Epithelial cells from the $\mathrm{CAM}$ ectoderm were observed to proliferate and move into the $\mathrm{PHB} / \mathrm{CHIT}$ component, forming fusion boundaries between the scaffold and CAM tissues, suggesting good biocompatibility and bioactivity of this biomaterial. Newly formed CAM tissue was observed also within the PHB/CHIT scaffold as the formation of CAM villi. These cells expressed smooth muscle actin, suggesting they were myofibroblasts. They were localized only in some areas around the implant. However, there was no excessive production of collagen I or extracellular matrix as revealed by WGA or pi- crosirius red staining (Fig. 5). CD68 staining did not identify the presence of macrophages.

\subsection{Angiogenesis within the scaffolds on the CAM}

Endothelial cell migration and sprouting are the most important steps of angiogenesis. We observed vessels leading to and from the implant on the surface of the CAM (Fig. 1), suggesting its vascularization after 5 days of implantation. This was confirmed by high-resolution videomicroscopy (Video S2${ }^{2}$ ) and ultrasound biomicroscopy (Video $\mathrm{S}^{3}{ }^{3}$ ). The presence of endothelial precursors and vessels inside the implant was confirmed by positive QH1 staining (Fig. 6) on scaffolds implanted into the quail chorioallantoic membrane.

\section{Discussion}

Cell adhesion to an implant and cell activity are influenced by properties of the implant surface such as surface tension, roughness,

\footnotetext{
2 In situ blood flow from the PHB/CHIT implanted scaffold including the vessels: doi:10.14573/altex.1807241s2

3 Blood flow inside of PHB/CHIT implanted scaffold by VeVo ultrasound system: doi:10.14573/altex.1807241s3
} 

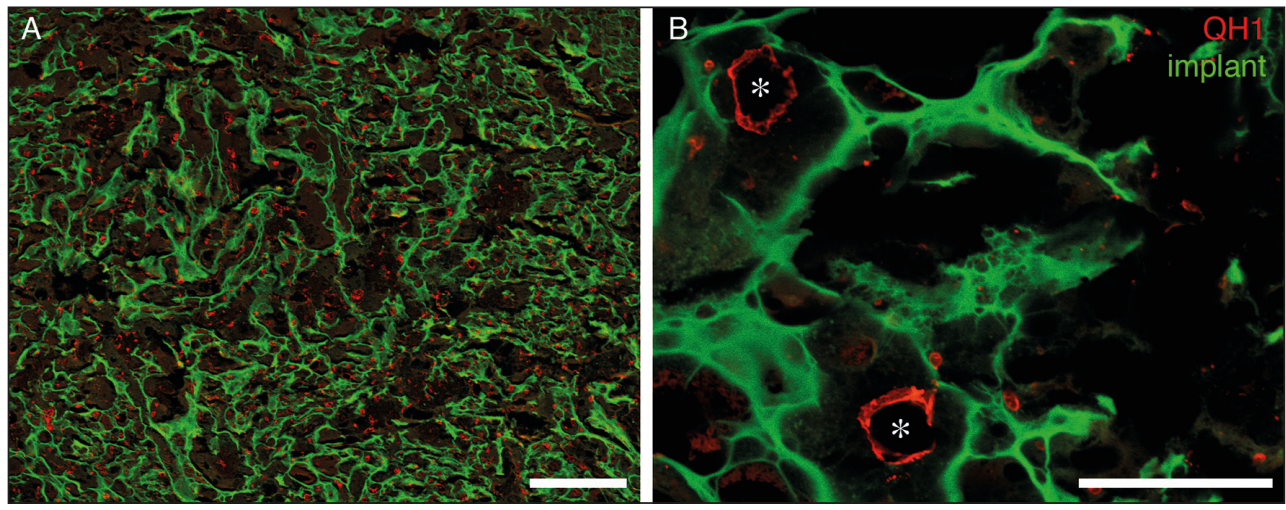

Fig. 6: Evidence of endothelial cells, hemangioblasts, and blood vessels inside the implanted scaffold

(A) QH1 positive endothelial cells; scale bar: $200 \mu \mathrm{m}$.

(B) Blood vessels (asterisk) and hemangioblasts are present inside of implanted scaffold; scale bar: $100 \mu \mathrm{m}$; biological replicates (9), technical replicates (72), for confirmatory images see1.

etc. (Wang et al., 2005). Chitosan provides a reservoir for the release of bioactive substances and also acts as a scaffold that fis amenable to colonization by cells (Barreto et al., 2016; Medvecky et al., 2014).

Polyhydroxybutyrate porous scaffolds in combination with biphasic calcium phosphate or chitosan are known to stimulate proliferation of fibroblasts and osteoblasts without inducing a proinflammatory response (Cool et al., 2007; Veleirinho et al., 2012; Tai et al., 2014). The average molecular weight of chitosan decreases after precipitation in propylene carbonate solution, but this does not appear to have negative effects as Giretova et al. (2016) found that lysozyme-degraded scaffolds containing a large fraction of low molecular weight chitosan (LMWC) were not cytotoxic even after 10 days of cultivation.

Previous studies have reported that various versions of chitosan-based scaffolds may support angiogenesis for different applications (Deng et al., 2010; Wang et al., 2012; Linn et al., 2003; Agarwal et al., 2016; Shahzadi et al., 2016); however only few studies have investigated this for the field of bone tissue engineering and these were only able to show evidence of angiogenesis on the scaffold surface (Pan et al., 2018; Ahmadi et al., 2010) or found that chitosan-acetate may actually inhibit angiogenesis (Shah et al., 2014). Others have attempted to enhance angiogenesis by loading chitosan scaffolds with recombinant vascular endothelial growth factor (VEGF) (Linn et al., 2003) or expressing VEGF with an adenoviral vector (Koç et al., 2014).

We here show clear evidence of blood vessel ingrowth into unseeded PHB/CHIT scaffolds without performing in vivo testing on animals and without expression or addition of VEGF. Using the QH1 antibody, we observed the presence of individual endothelial cells as well as vessels inside the porous biomaterial. However, there are also some QH1-positive cells not connected to the vessels. It is thus possible that hemangioblasts migrate into the implant and vasculogenesis takes place together with angiogenesis. This hypothesis would have to be tested by another experimental study.

We found that the chitosan implant surface has fibrotic effects, probably because the implant surface directly activates quiescent myofibroblasts to differentiate into myofibroblasts by providing a mechanical stimulus. Myofibroblasts are characterized by an excessive production of collagen (Majd et al., 2015). Using WGA labeling of extracellular matrix, which was found to be comparable with the established picrosirius red staining, we found that the fibrous layer was present around the implant outside of the layer of $\alpha$-smooth muscle actin ( $\alpha$-SMA) positive myofibroblasts (Fig. 4; Emde et al., 2014).

We did not find evidence of induction of a non-specific inflammatory reaction by the implant as we did not find an influx of CD68 positive macrophages. This may be because we put the implant on the CAM early during development (ED5), at a time when the immune system is still immature. Inflammatory angiogenesis, in which infiltrating macrophages are the source of angiogenic factors, has to be distinguished from direct angiogenic activity of the tested biomaterial with histological analysis and immunohistochemical staining for specific cell populations. It would be interesting to extend our studies also into the later period (Baiguera et al., 2012) to monitor myofibroblasts as they become apoptotic or fibrosis develops, and to observe the influx of macrophages as the immune system develops. However, in some countries protection of the avian embryo starts at ED12-14 when the nervous system develops sensory synapses and two thirds of gestation has elapsed.

The CAM was previously used as an in ovo method for evaluating the tissue response to various biomaterials, including composites based on collagen (Vargas et al., 2013), Elvax 40 (ethelyne-vinyl acetate copolymer; Langer and Folkman, 1976), hydron (poly-2-hydroxyethyl-methacrylate polymer; HydroMed; Ribatti et al., 1996), PCL (polycaprolactone; Singh et al., 2012), and matrix hydrogels (Fercana et al., 2017). This is a rather lowtech method, which makes it possible to continuously monitor angiogenesis, to easily and quickly obtain results, and to evaluate them in a relatively short time period. The response of the CAM to a biomaterial is similar to that of the mammalian animal model. However, in contrast to the mammalian model, it allows continuous monitoring, which makes it very attractive for rapid in ovol ex ovo angiogenesis evaluation (Magnaudeix et al., 2016; Jin et al., 2016). Angiogenesis in the CAM model is usually quantified by assessing the number of new vessels oriented towards the implant (Ribatti et al., 2006; Strick et al., 1991). This method is rather subjective and only considers angiogenesis outside or on the surface of the scaffold. The focus of our study was primarily to identify the presence of blood vessels in the internal structure of the scaffold.

Our results showed that the PHB/CHIT scaffold has satisfactory angiogenic properties and induces formation of CAM villi 
penetrating the pores of the implant. One can easily quantify the number of blood vessels in the vicinity of the implant, perform topographical and structural assessment using confocal or electron microscopy, and examine the cellular changes within the structure by immunohistochemistry.

The CAM model represents an intermediate step for testing biomaterial between the simple, in vitro model and the complex in vivo mammalian animal model (Valdes et al., 2002). Thus, it can reduce and replace animal experiments in the field of bone regenerative medicine.

\section{Conclusions}

Original porous $\mathrm{PHB} / \mathrm{CHIT}$ biomaterial designed for bone regeneration was tested for the first time with a short-term CAM assay. Previous studies monitored the pro-angiogenic properties and biocompatibility only on the surface of similar porous scaffolds based on chitosan. In this study, the methods were focused on monitoring of angiogenesis and biocompatibility inside of the scaffold, which provides more complex information for the qualitative assessment of the tested biomaterial. The methods allow observation of the surrounding CAM tissue reaction, presence of cells in the pores of the scaffold, and the comparison of vessels growing toward the implant with their actual presence inside it. The presence of hemangioblasts and endothelial cells inside the scaffold could be shown. It remains to be determined whether angiogenesis or vasculogenesis is involved. Myofibroblasts were found around the implant, but without excessive collagen deposition. This study confirmed that the CAM assay is a rapid, cost-effective and simple method to test and optimize new scaffolds before their use on larger experimental animals.

\section{References}

Agarwal, T., Narayan, R., Maji, S. et al. (2016). Gelatin/carboxymethyl chitosan based scaffolds for dermal tissue engineering applications. Int J Biol Macromol 93, 1499-1506. doi:10.1016/j.ijbiomac.2016.04.028

Ahmadi, R., Burns, A. J. and de Bruijn, J. D. (2010). Chitosan-based hydrogels do not induce angiogenesis. J Tissue Eng Regen Med 4, 309-315. doi:10.1002/term.247

Anderson, S. M., Siegman, S. N. and Segura, T. (2011). The effect of vascular endothelial growth factor (VEGF) presentation within fibrin matrices on endothelial cell branching. Biomaterials 32, 7432-7443. doi:10.1016/j.biomaterials.2011.06.027

Baiguera, S., Macchiarini, P. and Ribatti, D. (2012). Chorioallantoic membrane for in vivo investigation of tissue-engineered construct biocompatibility. J Biomed Mater Res Part B 100, 1425-1434. doi:10.1002/jbm.b.32653

Barreto, R. S., Quintans, J. S., Barreto, A. S. et al. (2016). Improvement of wound tissue repair by chitosan films containing (-)-borneol, a bicyclic monoterpene alcohol, in rats. Int Wound $J$ 13, 799-808. doi:10.1111/iwj.12385

Benes Jr., J., Melenovsky, V., Skaroupkova, P. et al. (2011). Myocardial morphological characteristics and proarrhythmic substrate in the rat model of heart failure due to chronic volume overload. Anat Rec 294, 102-111. doi:10.1002/ar.21280

Borges, J., Tegtmeier, F. T., Padron, N. T. et al. (2003). Chorioallantoic membrane angiogenesis model for tissue engineering: A new twist on a classic model. Tissue Eng 9, 441-450. doi:10.1089/107632703322066624

Brown, C. B., Drake, C. J. and Barnett, J. V.(1999). Antibodies directed against the chicken type II TGFbeta receptor identify endothelial cells in the developing chicken and quail. Dev Dyn 215, 79-85. doi:10.1002/(SICI)1097-0177(199905)215:1<79::AID-DVDY9 $>3.0 . \mathrm{CO} ; 2-\mathrm{H}$

Buríková, M., Bilčík, B., Máčajová, M. et al. (2016). Hypericin fluorescence kinetics in the presence of low density lipoproteins: Study on quail CAM assay for topical delivery. Gen Physiol Biophys 35, 459-468. doi:10.4149/gpb_2016014

Chavakis, E. and Dimmeler, S. (2002). Regulation of endothelial cell survival and apoptosis during angiogenesis. Arterioscler Thromb Vasc Biol 22, 887-893. doi:10.1161/01.ATV.0000017728.55907. A9

Concha, M., Vidal, A., Giacaman, A. et al. (2018). Aerogels made of chitosan and chondroitin sulfate at high degree of neutralization: Biological properties toward wound healing. J Biomed Mater Res B Appl Biomater 106, 2464-2471. doi:10.1002/jbm.b.34038

Cool, S. M., Kenny, B., Wu, A. et al. (2007). Poly(3 hydroxybutyrate-co-3-hydroxyvalerate) composite biomaterials for bone tissue regeneration: In vitro performance assessed by osteoblast proliferation, osteoclast adhesion and resorption, and macrophage proinflammatory response. J Biomed Mater Res 82, 599610. doi:10.1002/jbm.a.31174

Da-Lozzo, E. J., Moledo, R. C., Faraco, C. D. et al. (2013). Curcumin/xanthan-galactomannan hydrogels: Rheological analysis and biocompatibility. Carbohydr Polym 93, 279-284. doi:10.1016/j. carbpol.2012.02.036

Deng, C., Zhang, P., Vulesevic, B. et al. (2010). A collagen-chitosan hydrogel for endothelial differentiation and angiogenesis. Tissue Eng Part A 16, 3099-3109. doi:10.1089/ten.tea.2009.0504

Djonov, V., Schmid, M., Tschanz, S. A. and Burri, P. H. (2000). Intussusceptive angiogenesis: Its role in embryonic vascular network formation. Circ Res 86, 286-292. doi:10.1161/01. RES.86.3.286

Drake, C. J., Brandt, S. J., Trusk, T. C. and Little, C. D. (1997). TAL1/SCL is expressed in endothelial progenitor cells/angioblasts and defines a dorsal-to-ventral gradient of vasculogenesis. Dev Biol 192, 17-30. doi:10.1006/dbio.1997.8751

Emde, B., Heinen, A., Gödecke, A. and Bottermann, K. (2014). Wheat germ agglutinin staining as a suitable method for detection and quantification of fibrosis in cardiac tissue after myocardial infarction. Eur J Histochem 58, 2448. doi:10.4081/ ejh.2014.2448

Eun, J. P. and Koh, G. Y. (2004). Supression of angiogenesis by the plant alkaloid, sanguinarine. Biochem Biophys Res Commun 317, 618-624. doi:10.1016/j.bbrc.2004.03.077

Falkner, E., Eder, C., Kapeller, B. et al. (2004). The mandatory CAM testing of cells and scaffolds for tissue engineering: Benefits for the three Rs of cooperation with the vaccine industry. Altern Lab Anim 32, 573-580.

Fercana, G. R., Yemeni, S., Billaud, M. et al. (2017). Perivascular extracellular matrix hydrogels mimic native matrix microarchi- 
tecture and promote angiogenesis via basic fibroblast growth factor. Biomaterials 123, 142-154. doi:10.1016/j.biomaterials. 2017.01.037

Giles, P. B., Candy, C. L., Fleming, P. A. et al. (2005). VEGF directs newly gastrulated mesoderm to the endothelial lineage. Dev Biol 279, 169-178. doi:10.1016/j.ydbio.2004.12.011

Giretova, M., Medvecky, L., Stulajterova, R. et al. (2016). Effect of enzymatic degradation of chitosan in polyhydroxybutyrate/ chitosan/calcium phosphate composites on in vitro osteoblast response. J Mater Sci Mater Med 27, 181. doi:10.1007/s10856016-5801-7

Hazel, S. J. (2003). A novel early chorioallantoic membrane assay demonstrates quantitative and qualitative changes caused by antiangiogenic substances. J Lab Clin Med 141, 217-228. doi: $10.1067 / \mathrm{mlc} .2003 .19$

Jin, K., Ye, X., Li, S. et al. (2016). A biomimetic collagen/heparin multi-layered porous hydroxyapatite orbital implant for in vivo vascularization studies on the chicken chorioallantoic membrane. Graefes Arch Clin Exp Ophthalmol 254, 83-89. doi:10.1007/ s00417-015-3144-6

Junqueira, L. C., Bignolas, G. and Brentani, R. R. (1979). Picrosirius staining plus polarization microscopy, a specific method for collagen detection in tissue sections. Histochem J 11, 447-455. doi: 10.1007/BF01002772

Kang, M. S., Lee, N. H., Singh, R. K. et al. (2018). Nanocements produced from mesoporous bioactive glass nanoparticles. Biomaterials 162, 183-199. doi:10.1016/j.biomaterials.2018.02.005

Kant, R. J. and Coulombe, K. L. K. (2018). Integrated approaches to spatiotemporally directing angiogenesis in host and engineered tissues. Acta Biomater 69, 42-62. doi:10.1016/j.actbio. 2018.01.017

Klagsburn, M. and Moses, M. A. (1999). Molecular angiogenesis. Chem Biol 6, 217-224. doi:10.1016/S1074-5521(99)80081-7

Koç, A., Finkenzeller, G., Elçin, A. E. et al. (2014). Evaluation of adenoviral vascular endothelial growth factor-activated chitosan/ hydroxyapatite scaffold for engineering vascularized bone tissue using human osteoblasts: In vitro and in vivo studies. J Biomater Appl 29, 748-760. doi:10.1177/0885328214544769

Langer, R. and Folkman, J. (1976). Polymers for the sustained release of proteins and other macromolecules. Nature 263, 797800. doi: $10.1038 / 263797 \mathrm{a} 0$

Langer, R. and Vacanti, J. P. (1993). Tissue engineering. Science 260, 920-926. doi:10.1126/science.8493529

Linn, T., Erb, D., Schneider, D. et al. (2003). Polymers for induction of revascularization in the rat fascial flap: Application of vascular endothelial growth factor and pancreatic islet cells. Cell Transplant 12, 769-778. doi:10.3727/000000003108747244

Liu, X., Wang, X., Horii, A. et al. (2012). In vivo studies on angiogenic activity of two designer self-assembling peptide scaffold hydrogels in the chicken embryo chorioallantoic membrane. Nanoscale 4, 2720-2727. doi:10.1039/c2nr00001f

Magnaudeix, A., Usseglio, J., Lasgorceix, M. et al. (2016). Quantitative analysis of vascular colonisation and angio-conduction in porous silicon-substituted hydroxyapatite with various pore shapes in a chick chorioallantoic membrane (CAM) model. Acta Biomater 38, 179-189. doi:10.1016/j.actbio.2016.04.039

Majd, H., Scherer, S. S., Boo, S. et al. (2015). Novel micropatterns mechanically control fibrotic reactions at the surface of silicone implants. Biomaterials 54, 136-147. doi:10.1016/j.biomaterials. 2015.03.027

McQuinn, T. C., Bratoeva, M., deAlmeida, A. et al. (2007). High-frequency ultrasonographic imaging of avian cardiovascular development. Dev Dyn 236, 3503-3513. doi:10.1002/dvdy. 21357

Medvecky, L., Giretova, M. and Stulajterova, R. (2014). Properties and in vitro characterization of polyhydroxybutyrate-chitosan scaffolds prepared by modified precipitation method. J Mater Sci Mater Med 25, 777-789. doi:10.1007/s10856-013-5105-0

Moreno-Jiménez, I., Kanczler, J. M., Hulsart-Billstrom, G. et al. (2017). The chorioallantoic membrane assay for biomaterial testing in tissue engineering: A short-term in vivo preclinical model. Tissue Eng Part C Methods 23, 938-952. doi:10.1089/ ten.tec.2017.0186

Musumeci, G., Castrogiovanni, P., Leonardi, R. et al. (2014). New perspectives for articular cartilage repair treatment through tissue engineering: A contemporary review. World J Orthop 5, 80-88. doi:10.5312/wjo.v5.i2.80

Muzzarelli, R., Baldassarre, V., Conti, F. et al. (1988). Biological activity of chitosan. Ultrastructural study. Biomaterials 9, 247252. doi:10.1016/0142-9612(88)90092-0

Özcetin, A., Aigner, A. and Bakowsky, U. (2013). A chorioallantoic membrane model for the determination of anti-angiogenic effects of imatinib. Eur J Pharm Biopharm 85, 711-715. doi:10.1016/j. ejpb.2013.07.010

Pan, Y., Chen, J., Yu, Y. et al. (2018). Enhancement of BMP-2-mediated angiogenesis and osteogenesis by 2-N,6-O-sulfated chitosan in bone regeneration. Biomater Sci 6, 431-439. doi:10.1039/ C7BM01006K

Pandit, J., Sultana, Y. and Aqil, M. (2017). Chitosan-coated PLGA nanoparticles of bevacizumab as novel drug delivery to target retina: Optimization, characterization, and in vitro toxicity evaluation. Artif Cells Nanomed Biotechnol 45, 1397-1407. doi:10.1080/ 21691401.2016.1243545

Pardanaud, L., Altmann, C., Kitos, P. et al. (1987). Vasculogenesis in the early quail blastodisc as studied with a monoclonal antibody recognizing endothelial cells. Development 100, 339-349. http://dev.biologists.org/content/develop/100/2/339.full.pdf

Poonguzhali, R., Khaleel Basha, S. and Sugantha Kumari, V. (2018). Novel asymmetric chitosan/PVP/nanocellulose wound dressing: In vitro and in vivo evaluation. Int J Biol Macromol 112, 1300-1309. doi:10.1016/j.ijbiomac.2018.02.073

Rashidi, H. and Sottile, V. (2009). The chick embryo: Hatching a model for contemporary biomedical research. Bioessays 31, 459-465. doi:10.1002/bies.200800168

Ribatti, D., Vacca, A., Roncali, L. and Dommacco, F. (1996). The chick chorioallantoic membrane as a model for in vivo research on angiogenesis. Int J Dev Biol 40, 1189-1197. http://www.ijdb. ehu.es/web/paper.php?doi=9032025

Ribatti, D., Nico, B., Vacca, A. and Presta, M. (2006). The gelatin sponge - Chorioallantoic membrane assay. Nat Protoc 1, 85-91. doi:10.1038/nprot.2006.13

Ribatti, D. (2016). The chick embryo chorioallantoic membrane (CAM). A multifaceted experimental model. Mech Dev 141, 7077. doi:10.1016/j.mod.2016.05.003 
Sedmera, D. and McQuinn, T. (2008). Embryogenesis of the heart muscle. Heart Fail Clin 4, 235-245. doi:10.1016/j. hfc.2008.02.007

Shah, U. H., Gonjari, G. R. and Patil, A. E. (2014). Angiostatic property of chitosan acetatein the chick chorioallantoic membrane (CAM), in ovo. Bioscan 9, 1505-1508. http://www. thebioscan.in/Journals_PDF/9426\%20U\%20H\%20SAHA_ 3186.pdf

Shahzadi, L., Yar, M., Jamal, A. et al. (2016). Triethyl orthoformate covalently cross-linked chitosan-(poly vinyl) alcohol based biodegradable scaffolds with heparin-binding ability for promoting neovascularization. J Biomater Appl 31, 582-593. doi:10.1177/0885328216650125

Singh, S., Wu, B. M. and Dunn, J. C. (2012). Delivery of VEGF using collagen-coated polycaprolactone scaffolds stimulates angiogenesis. J Biomed Mater Res A 100, 720-727. doi:10.1002/ jbm.a.34010

Steffens, L., Wenger, A., Stark, G. B. and Finkenzeller, G. (2009). In vivo engineering of a human vasculature for bone tissue engineering applications. J Cell Mol Med 13, 3380-3386. doi: 10.1111/j.1582-4934.2008.00418.x

Stegen, S., van Gastel, N. and Carmeliet, G. (2015). Bringing new life to damaged bone: The importance of angiogenesis in bone repair and regeneration. Bone 70, 19-27. doi:10.1016/j.bone. 2014.09.017

Stocum, D. L. (2002). Regenerative biology and medicine. J Musculoskelet Neuronal Interact 2, 270-273. http://www.ismni.org/ jmni/pdf/7/Stocum.pdf

Strick, D. M., Waycaster, R. L., Montani, J. P. et al. (1991). Morphometric measurements of chorioallantoic membrane vascularity: Effects of hypoxia and hyperoxia. Am J Physiol 260, H13851389. doi:10.1152/ajpheart.1991.260.4.H1385

Tai, H. Y., Fu, E., Cheng, L. P. and Don, T. M. (2014). Fabrication of asymmetric membranes from polyhydroxybutyrate and biphasic calcium phosphate/chitosan for guided bone regeneration. $J$ Polym Res 21, 421. doi:10.1007/s10965-014-0421-8

Tan, M. L., Shao, P., Friedhuber, A. M. et al. (2014). The potential role of free chitosan in bone trauma and bone cancer management. Biomaterials 35, 7828-7838. doi:10.1016/j.biomaterials.2014.05.087

Tay, S. M., Heng, P. S. and Chan, L. W. (2012). The chick chorioallantoic membrane imaging method as a platform to evaluate vasoactivity and assess irritancy of compounds. J Pharm Pharmacol 64, 1128-1137. doi:10.1111/j.2042-7158.2012.01506.x

Tomco, M., Petrovova, E., Giretova, M. et al. (2017). In vitro and in vivo study of microporous ceramics using MC3T3 cells, CAM assay and a pig animal model. Anat Sci Int 92, 569-580. doi:10.1007/s12565-016-0362-x

Valdes, T. I., Kreutzer, D. and Moussy, F. (2002). The chick chorioallantoic membrane as a novel in vivo model for the testing of biomaterials. J Biomed Mater Res 62, 273-282. doi:10.1002/ jbm.10152

Vargas, G. E., Durand, L. A., Cadena, V. et al. (2013). Effect of nano-sized bioactive glass particles on the angiogenic properties of collagen based composites. J Mater Sci Mater Med 24, 12611269. doi:10.1007/s10856-013-4892-7

Veleirinho, B., Coelho, D. S., Dias, P. F. et al. (2012). Nanofibrous poly(3-hydroxybutyrate-co-3-hydroxyvalerate)/chitosan scaffolds for skin regeneration. Int J Biol Macromol 51, 343-350. doi:10.1016/j.ijbiomac.2012.05.023

Wang, X., Li, Q., Hu, X. et al. (2012). Fabrication and characterization of poly(L-lactide-co-glycolide) knitted mesh-reinforced collagen-chitosan hybrid scaffolds for dermal tissue engineering. J Mech Behav Biomed Mater 8, 204-215. doi:10.1016/j. jmbbm.2012.01.001

Wang, Y. W., Yang, F., Wu, Q. et al. (2005). Effect of composition of poly(3-hydroxybutyrate-co-3- hydroxyhexanoate) on growth of fibroblast and osteoblast. Biomaterials 26, 755-761. doi:10.1016/j.biomaterials.2004.03.023

Yu, W. L., Sun, T. W., Qi,C. et al. (2017). Enhanced osteogenesis and angiogenesis by mesoporous hydroxyapatite microspheres-derived simvastatin sustained release system for superior bone regeneration. Sci Rep 7, 44129. doi:10.1038/srep44129

Zhang, J., Deng, A., Yang, Y. et al. (2015). HPLC detection of loss rate and cell migration of HUVECs in a proanthocyanidin cross-linked recombinant human collagen-peptide (RHC)-chitosan scaffold. Mater Sci Eng C Mater Biol Appl 56, 555-563. doi:10.1016/j.msec.2015.07.019

\section{Conflict of interest}

There is no conflict of interest.

\section{Acknowledgements}

The present study was supported in part by the Grant Agency of Ministry of the Education, Science, Research and Sport of the Slovak Republic no. VEGA-1/0046/16; Slovak Research and Development Agency under the contract no. APVV-17-0110; Ministry of Education, Youth and Sports of the Czech Republic PROGRESS-Q38, Charles University SVV Graduate Student Training Program; Ministry of Health AZV CR, 15-32497A, 1728265A; Czech Academy of Sciences RVO: 67985823, INTERCOST LTC17023, and LM2015062 Czech-BioImaging CZ.02. 1.01/0.0/0.0/16_013/0001775 Modernization and support of research activities of the national infrastructure for biological and medical imaging Czech-BioImaging funded by OP RDE.

The authors would like to thank Blanka Topinková and Mariana Saulicova from the Institute of Anatomy, Charles University in Prague, Czech Republic and the Institute of Anatomy, University of Veterinary Medicine and Pharmacy in Kosice, Slovak Republic, for their laboratory facilities. 